\title{
Metamemoria y estrategias mnémicas en escolares
}

\author{
JOSÉ M. DÍAZ \\ MARÍA JOSÉ RODRIGO \\ Universidad de La Laguna
}

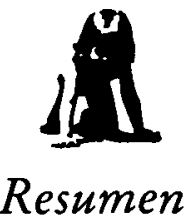

Este articulo se propone determinar la relación entre el grado de metamemoria alcanzado en niños de 7 a 14 años, y su rendimiento en dos pruebas de memoria. Estas se diseñaron de forma que midiesen no sólo el recuerdo, sino también el empleo de dos estrategias mnémicas: la repetición y el agrupamiento. Los resultados respecto a la primera indican un mejor rendimiento de los sujetos altos en metamemoria, sólo dentro del grupo de 7 años. En cuanto a la estrategia de agrupamiento, parece que su empleo no está asociado necesariamente a un mejor rendimiento. Asi, en los niños mayores, son los bajos en metamemoria los que más la utilizan, sin que su recuerdo sea superior al del grupo de los altos. Entre los niños de 7 años los resultados son parecidos, de manera que los altos en metamemoria recuerdan más, pero no emplean con más frecuencia dicha estrategia.

Palabras clave: Metamemoria, Estrategias mnémicas, Rendimiento mnémico.

\section{Metamemory and mnemonic strategies in school children}

\section{Abstract}

The present paper is an attempt to determine the relationship between the level of metamemory attained by children aged 7 and 14 , and their performance in two memory tasks. These tasks were designed to measure not only memory but also the use of two mnemonic strategies: rebearsal and clasification. The results of the first task show a better performance by subjects with a bigh level of metamemory, but only within the group of children aged 7 . In the case of the clasification strategy, it seems that its use is not necessarily linked to better performance. Thus, in the group of older children, those with a low level of metamemory make more use of the latter strategy, though their memory is not higher than that of the children with a bigh level of metamemory. In the group of children aged 7 , the results are similar: those with a bigh level of metamemory have better recall, but do not use the clasification strategy more frequently.

Keywords: Metamemory, Mnemonic strategies, Mnemonic performance.

Dirección del autor: Universidad de La Laguna, Departamento de Psicología Cognitiva, 38071, La Laguna, Santa Cruz de Tenerife.

Original recibido: Diciembre 1988. Revisión recibida: Marzo 1989. Aceptado: Marzo 1989. 
Una de las razones que explican el interés despertado por el estudio de la metamemoria ha sido su posible conexión funcional con el rendimiento mnémico (Hagen, 1975; Brown, 1978; Lunzer, 1979). El presupuesto teórico de partida de estas investigaciones es que, en general, el autoconocimiento de las propiedades y modo de funcionamiento del sistema cognitivo es un factor importante que determina un rendimiento eficaz de éste (Borkovsky y Cavanaugh, 1981).

Sin embargo, cuando se examinan con cierto detenimiento los resultados obtenidos sobre el efecto de la metamemoria en el rendimento mnémico, observamos que no son tan nítidos como sería deseable. En primer lugar, se echa en falta un modelo teórico que explique las conexiones entre la metamemoria y la memoria. Este modelo debería tener en cuenta la posible interferencia en dicha relación de variables ajenas a la metamemoria y que pueden influir en el rendimiento mnémico, hasta el punto de eclipsar los efectos de ésta. Los propios cambios evolutivos en la capacidad estructural de la memoria o en la velocidad de procesamiento de la información serían dos ejemplos de estas variables.

No obstante, los modelos existentes (Flavell, 1981; Herrman, 1982) presentan a este respecto un elevado grado de imprecisión, y en general parecen haber despertado escaso interés entre los investigadores, que han realizado sus estudios con notable independencia de los mismos.

Fruto directo de ello ha sido la disparidad de enfoques y de resultados que se han acumulado en la literatura, hasta el punto de que incluso los trabajos de revisión dibujan panoramas distintos entre sí. Entre estos trabajos de revisión merece destacarse, a nuestro juicio, el de Schneider (1985), no sólo por la amplitud de las investigaciones que recoge, sino también por la aplicación del procedimiento de meta-análisis (Glass, 1976) que permite un resumen cuantificado de los hallazgos empíricos acerca de un tema de investigación.

De este trabajo se desprende que los resultados obtenidos sobre la conexión funcional de la metamemoria y la memoria no son tan desalentadores como apuntan autores como Cavanaugh y Perlmutter (1982). Así, se ha demostrado, por ejemplo, que la metamemoria es mejor predictor del rendimiento mnémico que la inteligencia o el estilo cognitivo (Borkowski et al., 1983).

Pero más allá de resultados particulares como éste, lo más relevante de la revisión de Schneider es que, una vez que los hallazgos de las distintas investigaciones se transforman en índices de correlación -mediante el meta análisis-, el valor medio que toman es 0,41 , considerablemente superior al que generalmente se daba por existente entre la metamemoria y la memoria: «La integración cuantitativa de los hallazgos empíricos indica efectivamente que la metamemoria está relacionada de forma sustancial con el comportamiento y el rendimiento mnémicos" (Schneider, 1985, p. 98).

Ahora bien, esta valoración global no puede hacernos pasar por alto la gran diversidad encontrada por Schneider entre los resultados de las investigaciones revisadas. Dicha disparidad obedece fundamentalmente a dos razones, la primera de naturaleza teórica y la segunda de carácter metodológico. Los modelos teóricos citados anteriormente, que intentan explicar la relación entre la metamemoria y la memoria no aportan la claridad ni ri- 
queza conceptuales que serían deseables. Por ello no han dado lugar a hipótesis explicativas sobre la naturaleza de dicha relación.

La segunda razón es la diversidad de procedimientos metodológicos utilizados para evaluar la metamemoria. Así, Schneider (1985) ha encontrado en su revisión que los métodos de evaluación de la metamemoria influyen en los resultados obtenidos sobre su conexión con la memoria. Aquellos estudios que no han podido establecer una relación clara entre ambos sistemas han empleado por lo general entrevistas y cuestionarios, que son las medidas tradicionales de carácter verbal de la metamemoria. Por el contrario, cuando se utilizan otras formas de evaluación, como por ejemplo la predicción del propio rendimiento, el juicio sobre una situación de memorización presentada gráficamente, o la disponibilidad para el recuerdo, entonces los lazos entre la metamemoria y la memoria aparecen mejor definidos. (Se puede consultar al respecto la revisión de Cavanaugh y Perlmutter, 1982.)

Además de estos factores generales de índole conceptual y metodológica, Schneider (1985) ha constatado los efectos de tres variables criticas sobre la relación metamemoria-memoria.

En primer lugar, una fuente de variaciones en los resultados es el nivel de dificultad de la tarea de memoria que se utilice. Cuando ésta requiere el empleo de estrategias más o menos elaboradas, como la clasificación de los ítems, la relación con la metamemoria no es tan apreciable como sucede cuando la tarea es más sencilla, como, por ejemplo, recordar un conjunto pequeño de ítems (Cavanaugh y Perlmutter, 1982; Wellman, 1983).

En segundo lugar, se observan los efectos de la edad de los sujetos que intervienen en las investigaciones. De forma regular, a medida que ésta se incrementa, la relación metamemoria-memoria se dibuja con más claridad (Wellman, 1977; Brown y Lawton, 1977).

Por último, hay que considerar el componente de metamemoria que esté en juego (según la clasificación de Flavell y Wellman, 1977). Así, cuando intervienen variables de "persona», como la predicción del rendimiento mnémico (Levin et al., 1977; Yussen y Berman, 1981), se constata una relación más clara con la memoria que cuando lo hacen variables de "tarea", como el número de ítems a recordar, o las relaciones conceptuales entre ellos (Cavanaugh y Borkowski, 1980) o variables del componente "estrategia como, por ejemplo, la probabilidad de agrupamiento de ítems de distintas categorías conceptuales (Wimmer y Tornquist, 1980).

Ahora bien, hemos de señalar que los efectos de esta variable -componente de metamemoria - interactúan con los del método de medida empleado: «Hay buenas razones para creer que los poco convincentes resultados de los estudios sobre metamemoria relacionados con las estrategias organizativas se deben, al menos en parte, al método de evaluación que se ha empleado [la entrevista].» (Schneider, 1985. p. 100).

Teniendo en cuenta estos resultados, en la presente investigación optamos, en primer lugar, por la elaboración de un instrumento de evaluación de la metamemoria de carácter gráfico-verbal que permitiera una evaluación más objetiva de los conocimientos metamnémicos. El procedimiento requiere que los niños realicen un juicio comparativo entre dos condiciones de memorización (favorable y desfavorable) y ofrezcan una explicación acerca de ese juicio. Aunque se trata de una tarea con un innegable com- 
ponente introspectivo, la explicación hace referencia a un aspecto particular del conocimiento metamnémico y se realiza inmediatamente después del juicio, dos condiciones éstas que garantizan una mayor objetividad en el autoinforme (Ericson y Simon, 1980).

Por otra parte, en una investigación previa (Díaz y Rodrigo, en prensa) habíamos comprobado la estrecha relación existente entre el nivel de metaconocimiento de diversas variables contextuales y de tarea que afectan a la memorización, y el nivel de recuerdo en ciertas tareas. Los componentes de metamemoria (v. gr., interferencia atencional, ruido, implicación emocional, familiaridad, presencia de frases-palabras, efectividad de estrategias mnémicas de agrupamiento y repetición) se evaluaron mediante técnicas gráfico-verbales como la que acabamos de describir, mientras que las pruebas de memoria reproducían fielmente en condiciones reales las situaciones de memorización a la que se hacía referencia en la evaluación de la metamemoria. La única excepción fueron las estrategias de agrupamiento y repetición, que por su naturaleza no se prestaban a una manipulación directa en las pruebas de recuerdo. Ello motivó la realización de la presente investigación, en la que pretendemos estudiar la influencia del conocimiento metamnémico global -evaluado mediante las mismas técnicas gráfico-verbales- en la utilización de dichas estrategias en tareas de memorización.

En cuanto a la estrategia de agrupamiento o categorización, algunos estudios (Kobasigawa, 1977; Lange, 1978) indican que hay un rango de edades (de los 6 a los 10 años) en el que los niños, si bien reconocen en el material que estudian los beneficios de su categorización, no emplean esta estrategia (al menos espontáneamente), cosa que sí hacen los niños mayores. Ello ha llevado a los investigadores a centrar su interés en aquellos aspectos de la tarea de memorización - v. gr., las instrucciones o el tipo de relación entre los ítems- que pudieran fomentar el empleo espontáneo de esta estrategia en edades más tempranas, considerando que la enseñanza de la misma no se va a traducir necesariamente en un aumento de su frecuencia de empleo.

Por lo que respecta a la estrategia de repetición, es importante diferenciar la repetición pasiva o primaria (Bjork, 1975) de la repetición-elaboración. La primera consiste en el mantenimiento de la información en la memoria a corto plazo, y está asociada a la continua vocalización o sub-vocalización de los ítems, que se pierden al cesar ésta. Por el contrario, la repetición-elaboración lleva aparejado un procesamiento más profundo de la información (Craik y Lockhart, 1972), para categorizar el nuevo material, formar imágenes mentales $y$, en general, integrarlo en la memoria a largo plazo, lo que se traduce en su disponibilidad mucho tiempo después de su memorización (Baine, 1986).

Nuestro propósito era determinar si el nivel de conocimiento metamnémico influye, por una parte, en el rendimiento en las tareas de memorización, y, por otra, en la probabilidad de emplear las estrategias que acabamos de describir. Para ello se hacia necesario disponer de indicadores de utilización de las estrategias de agrupamiento y de repetición que fueran independientes de la cantidad de recuerdo. La elección de un índice de agrupamiento no plantea muchos problemas, ya que en la literatura se pueden encontrar varios. Nos inclinamos por el empleo de la ARC (Adjusted Ratio of Clustering) dadas sus ventajas (Roenker, Thompson y Brown, 1971). 
Este índice evalúa, en una escala de cero a uno, el orden en que se recuerdan los ítems de distintas categorías (fijadas de antemano) que el sujeto ha recibido de forma entremezclada, $y$ es independiente de la cantidad total de ítems recordados.

Encontrar un indicador del empleo de la estrategia de repetición (activa o pasiva) era más complejo, sobre todo si se pretende evitar el uso de procedimientos introspectivos. Para superar esta limitación nos inspiramos en el trabajo de Bellezza y. Walker (1974), que a pesar de situarse en un contexto teórico distinto, tiene un planteamiento interesante para nuestros propósitos. En su investigación, estos autores demostraron que si los sujetos sabían que, además de una prueba de recuerdo inmediato, iban a pasar otra de recuerdo diferido, entonces dedicaban parte de la capacidad de la memoria a corto plazo a "trasvasar» la información de la memoria a largo plazo. Esto supone que su recuerdo inmediato no era tan bueno como el de los sujetos que ignoraban la realización de esa segunda prueba diferida. En cambio, estos últimos obtenían en la prueba de recuerdo diferido un rendimiento muy inferior al de los sujetos del primer grupo, que conocían la existencia de las dos pruebas.

Ambas condiciones experimentales requieren, pues, diferentes estrategias de repetición. Para memorizar desde una perspectiva de recuperación inmediata de la información, es apropiado el simple empleo de estrategias repetitivas de mantenimiento. Por el contrario, para retener las mismas unidades de información durante un tiempo mayor, es pertinente el empleo de estrategias de repetición activa o elaboración. Siguiendo la lógica de este paradigma experimental, la observación de pautas de recuerdo diferenciales como las arriba descritas sería un claro índice de la utilización de uno u otro tipo de estrategia. Si esta pauta diferencial en las pruebas de memoria va asociada a un cierto nivel de conocimiento metamnémico, podría deducirse que éste facilita una evaluación precisa de las demandas de la tarea, guiando el empleo de una u otra estrategia de repetición.

\section{METODO}

\section{Sujetos}

Participaron 20 alumnos de segundo, y 20 de octavo de EGB del CNP, Anejo a la Escuela de Magisterio de La Laguna. Para su selección se administró la prueba de metamemoria (cuya descripción aparece más abajo) a 154 sujetos, de los que se escogieron en cada uno de los dos cursos a los que obtuvieron puntuaciones extremas: los 10 más altos y los diez más bajos.

\section{Diseño}

Para la estrategia de agrupamiento, la variable independiente fue el grupo de metamemoria (alto o bajo), y las variables dependientes fueron el rendimiento mnémico (cantida de ítems recordados) y el grado de agrupamiento.

Con respecto a la estrategia de repetición, se incluía otra variable independiente, de naturaleza experimental (además del grupo de metamemo- 
ria): el tipo de instrucción. Constaba de dos niveles: estar o no avisado de la existencia de una prueba de recuerdo diferido. La variable dependiente era el rendimiento mnémico.

\section{Instrumentos}

Para evaluar la metamemoria se emplearon dos cuadernillos, elaborados expresamente por los investigadores. En cada una de sus páginas se presentaba gráficamente una situación de la vida cotidiana de dos niños en la que estaba implicada una condición de memorización. Tenían la estructura que puede verse en la Figura 1: a la izquierda un dibujo que introducía la pe-

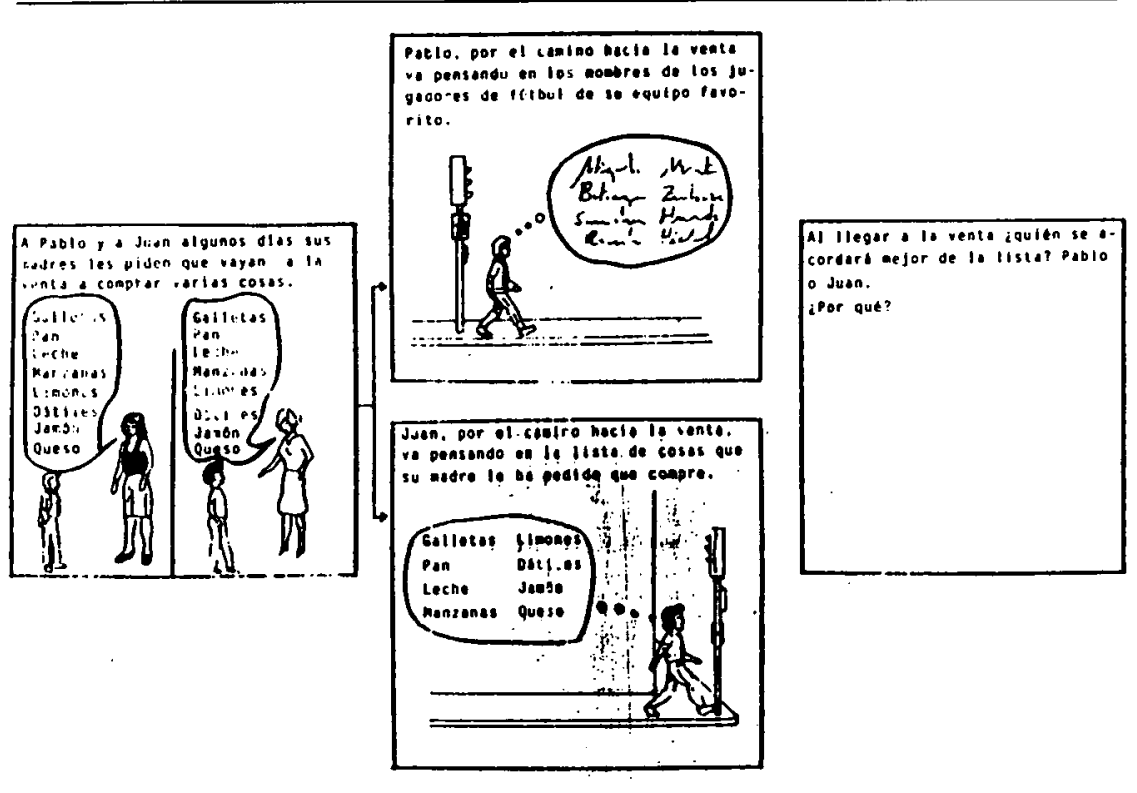

Modelo de item de la prueba de metamemoria

queña historia; en el centro, dos viñetas, en cada una de las cuales un personaje memorizaba en una determinada condición (favorable o desfavorable) para cumplir algún objetivo. Se procuró que la memorización no fuera un ejercicio inútil, sino que tuviera sentido en relación con la meta que los personajes debían alcanzar. A la derecha de la hoja aparecía un recuadro con la pregunta crítica sobre cuál de los dos protagonistas tendría más éxito en su tarea de memorización. A continuación los niños debían explicar brevemente las razones que les llevaban a tomar su decisión.

Los dos cuadernillos presentaban las mismas condiciones de memorización, si bien diferían en detalles tales como el lugar en que se desarrollaba la acción, o que fuese el padre o la madre del personaje el que le solicitase la tarea en cuestión. Se controlaban así los efectos extraños que pudiesen estar asociados a tales rasgos irrelevantes del instrumento.

En cuanto a la memoria, se emplearon dos pruebas. En la parte de la 
investigación centrada en la estrategia de agrupamiento, los sujetos recibían una hoja con 20 dibujos que representaban objetos pertenecientes a cuatro categorías naturales (escogidos de forma que su familiaridad fuese homogénea). Para la estrategia de repetición-codificación se utilizaron cuatro listas presentadas de forma auditiva, cada una con cinco items de parecida familiaridad.

\section{Procedimiento}

Como ya hemos indicado, en un paso previo a la investigación se administró la prueba de metamemoria a un conjunto numeroso de niños de $2 .^{\circ}$ y $8 .^{\circ}$ de EGB (medias de 7,5 y 13,8 años, respectivamente) para proceder a seleccionar, dentro de cada edad, a los 20 sujetos extremos (10 altos y 10 bajos).

Estos sujetos recibieron las pruebas de memoria en dos sesiones: en la primera la correspondiente a la estrategia de categorización, y en la segunda la de la estrategia de repetición. En ambos casos se les informaba de que al final de las pruebas recibirían un premio, y que éste sería mayor o menor en función de la cantidad de ítems recordados. Dada la edad de los alumnos de segundo de EGB, los premios que podían alcanzar se habían dibujado sobre una escala que indicaba los puntos necesarios para obtener cada uno. Los premios y las escalas eran diferentes para segundo y para octavo. Todo ello estaba encaminado a interesar a los sujetos en la tarea.

Con respecto a la estrategia de repetición, las instrucciones que los sujetos recibían estaban en función del grupo experimental al que perteneciesen. La mitad de los niños de cada curso no era avisada de la existencia de una prueba de recuerdo diferido (al final de todas las listas) mientras que la otra mitad de los niños sí lo sabía desde el primer momento, como también sabía que cada item recordado en esa segunda prueba valdría más puntos que los recordados inmediatamente después de cada lista. Se seguía de esta forma el procedimiento experimental de Bellezza y Walker (1974).

\section{RESULTADOS}

\section{Estrategia de repetición-codificación}

En primer lugar se realizó un análisis de varianza $2 \times 2 \times 2 \times 2$, con las variables independientes curso, tipo de instrucción (avisado vs. no avisado), grupo de metamemoria (alto vs. bajo) y momento (recuerdo inmediato vs. final), ésta última con medidas repetidas. La variable dependiente era la cantidad de recuerdo.

La interacción de mayor orden que resultó significativa fue Curso $\times$ Tipo de instrucción $\times$ Momento $(F(1,28=4,46 ; p<0,05)$. En la Figura 2 puede apreciarse el comportamiento de la variable dependiente. Lo que destaca en primer lugar es la diferencia en las pautas de recuerdo de los sujetos de segundo frente a los de octavo. En los del curso inferior se registran resultados similares a los encontrados por Bellezza y Walker (1974) en adultos, aunque algo menos nítidos. Los sujetos avisados recuerdan menos en las pruebas inmediatas que los no avisados, invirtiéndose la diferencia para el recuerdo diferido. En cambio, en octavo de EGB la tendencia no está tan clara, de forma que en los recuerdos inmediatos los niños avisados al- 
FIGURA 2

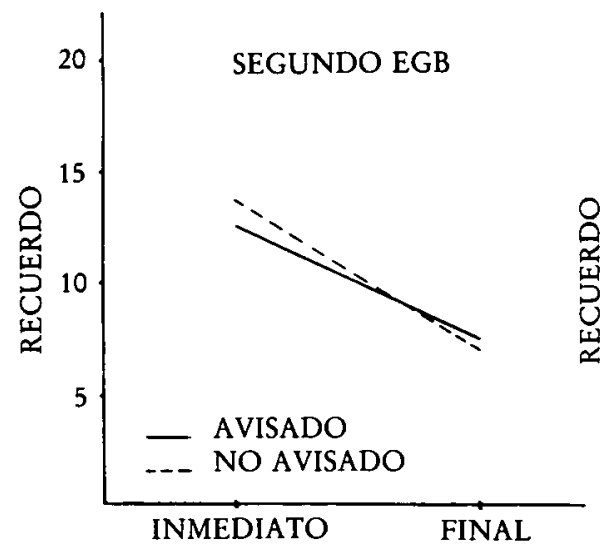

MOMENTO

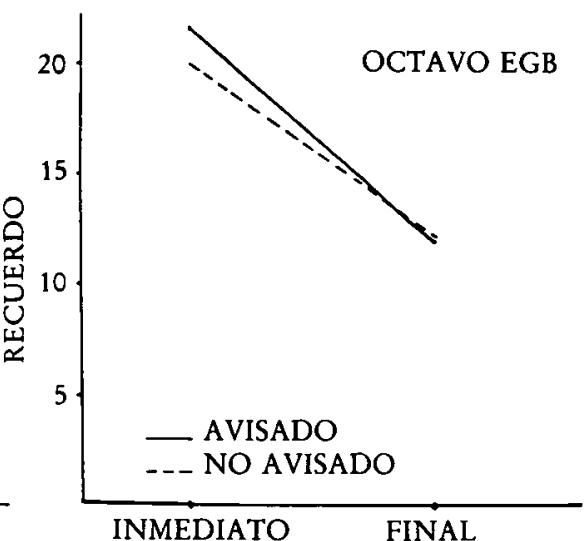

MOMENTO

Efectos del curso, momento y tipo de instrucción sobre el recuerdo

canzan mejores resultados, y en el recuerdo final las diferencias entre ambos grupos son prácticamente imperceptibles.

Por otra parte, debemos señalar el efecto significativo que tuvo la interacción de las variables Curso y Grupo de metamemoria $(F(1,28)=6,10$; $p=0,02$ ) sobre el recuerdo, y que queda reflejado en la Figura 3 . En ella puede observarse cómo las diferencias en el recuerdo entre los sujetos altos y bajos en metamemoria están mucho mejor definidas en segundo que en octavo, donde apenas si existen.

El distinto comportamiento de los alumnos de un curso y otro nos llevó a repetir este Anova, pero ahora de forma independiente para cada curso.

Como era de esperar, comprobamos que los efectos de las variables independientes no eran los mismos en ambos casos. Mientras que en octavo no se encontró ningún efecto significativo sobre el recuerdo, sí los hubo

FIGURA 3

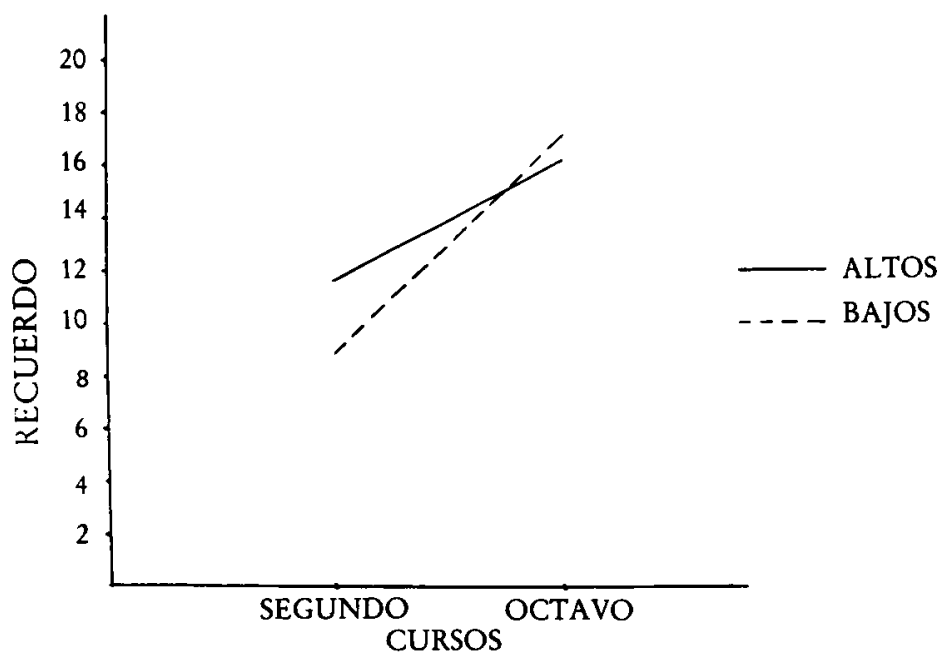

Efectos del curso y del grupo de meta-memoria sobre el recuerdo (Tarea 1) 


\section{1}

FIGURA 4

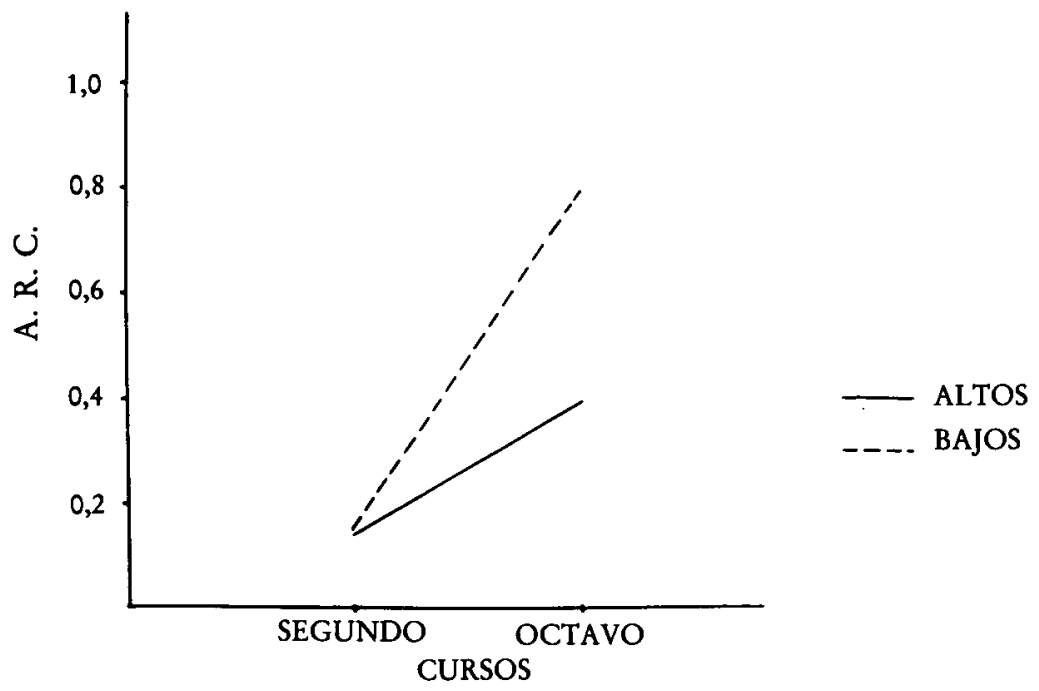

Efectos del curso y del grupo de meta-memoria sobre el plan de agrupamiento

en segundo. En este curso, los niños altos en metamemoria tienen un recuerdo significaticamente superior al de los bajos $(F(1,14)=11,4$; $\mathrm{p}<0,05)$.

\section{Estrategia de agrupamiento}

Como se recordará, con respecto a esta estrategia estábamos interesados en determinar fundamentalmente dos extremos. Uno era la relación de la meta-memoria con la cantidad de recuerdo, y el otro su relación con el grado de agrupamiento.

Para ello se realizó un análisis multivariado de la varianza (MANOVA), con las variables Grupo de metamemoria (alto vs. bajo) y Curso como in-

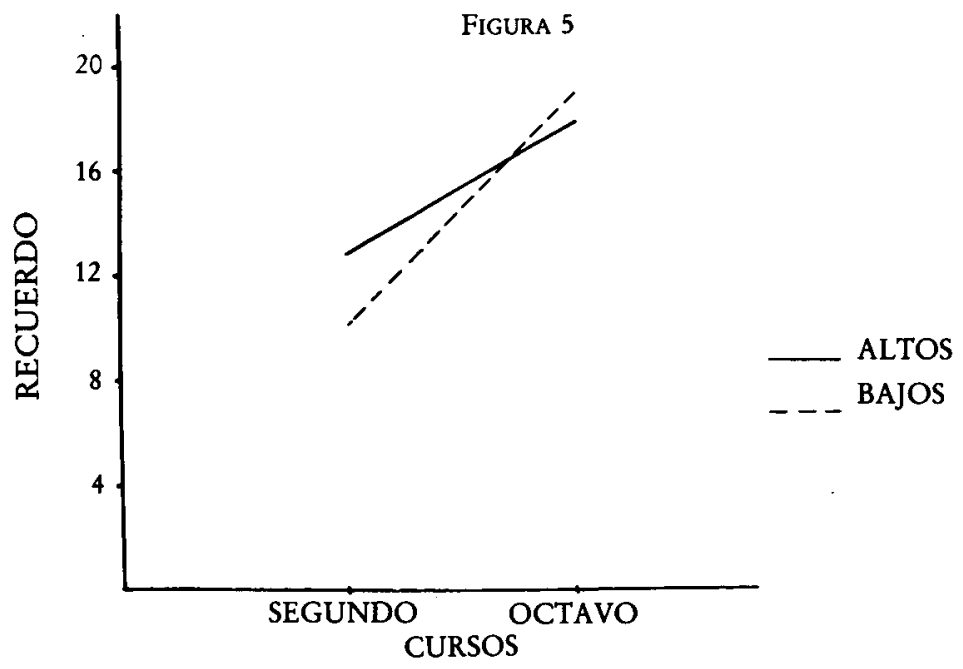


dependientes, y la cantidad de recuerdo y el grado de agrupamiento como variables dependientes. Hubo un efecto significativo debido a la interacción Curso $\times$ Grupo de metamemoria, sobre ambas variables dependientes $(T=7,43 ; \mathrm{p}<0,05)$. El significado de esta interacción para cada variable dependiente aparece en las Figuras 4 y 5 . La primera corresponde al grado de agrupamiento; en ella se observa que sólo en octavo de EGB hay diferencias entre los sujetos altos y los bajos en metamemoria, de forma que estos últimos registran un grado de agrupamiento notablemente superior al de los sujetos altos.

La Figura 5, referente a la otra variable dependiente, el recuerdo, muestra que en segundo de EGB los niños altos en metamemoria tienen mejor rendimiento mnémico que los bajos, lo que no sucede en octavo, donde la diferencia entre ambos grupos es mínima.

\section{DISCUSION}

Con respecto a la estrategia de repetición, nuestros resultados indican la existencia de pautas diferentes en el recuerdo inmediato y diferido según la edad de los sujetos. Para los alumnos de segundo de EGB hay más claridad en los datos que para los de octavo.

Para los primeros se ha podido constatar una pauta de respuesta similar a la encontrada por Bellezza y Walker (1974) con sujetos adultos. Los niños de esta edad con un nivel alto de metamemoria distribuyen su capacidad mnémica entre el recuerdo inmediato y el almacenamiento para un recuerdo posterior, en función de las demandas de la tarea. En cambio, los de baja metamemoria no desarrollan esta pauta diferencial (ya sea por falta de sensibilidad o por no ser capaces de activar adecuadamente sus recursos mnémicos).

Por otra parte, la metamemoria afecta directamente al grado de recuerdo alcanzado, que resulta superior para los niños altos en dicha variable que para los bajos.

En cambio, en octavo de EGB los resultados no son tan claros. Una posible explicación a este hecho es que el cuestionario que evalúa la metamemoria no discrimina adecuadamente entre los sujetos bajos y los altos. Este efecto techo sitúa a ambos grupos extremos en la zona superior de la escala, por lo que no es suficiente la distancia entre ellos. Para intentar solventar esta dificultad se podría atender, más que a la puntuación global del cuestionario, a la obtenida en aquellos ítems relativos específicamente a las estrategias mnémicas. Sin embargo, esta medida parcial sería menos fiable. Por otra parte, en investigaciones previas de estos autores (Díaz y Rodrigo, en prensa) se ha constatado que entre las variables que afectan al recuerdo, las estrategias mnémicas de repetición y codificación son muy bien conocidas, por lo que cabe esperar que su grado de conocimiento esté reflejado de manera ajustada en una puntuación global de metamemoria.

De otro lado, no se puede descartar el que las cargas de memoria empleadas no hayan sido adecuadas. La escasa cantidad de ítems a recordar y su familiaridad pueden haber contribuido a que para la mayoría de los niños de esta edad la tarea haya sido muy fácil, por lo que no habrían tenido necesidad de emplear a fondo sus recursos mnémicos. En este sentido, Brown 
(1980) señala la importancia del nivel de dificultad de la tarea a la hora de poner en juego el conocimiento metacognitivo.

La ausencia de resultados nítidos en octavo curso se ha repetido en el caso de la estrategia de agrupamiento, lo cual parece apoyar la línea argumental que hemos apuntado. Nuestros resultados, para esta edad, indican que la relación entre la metamemoria y la memoria es la inversa de la que cabría esperar en principio. En efecto, los niños con bajo nivel de metamemoria emplean más la estrategia de agrupamiento, pero ello no les conduce a un incremento significativo en el recuerdo. En cualquier caso, no debemos olvidar que la discriminación entre los sujetos altos y los bajos no es tan clara como sería deseable. Ha de señalarse, adicionalmente, el efecto que puede haber tenido el que los estímulos se presentasen en forma gráfica, con lo que ello significa en cuanto a facilidad de aprendizaje (Postman, 1978).

Por lo que respecta a segundo curso, hemos podido comprobar que la metamemoria guarda una relación más estrecha con el recuerdo que con el grado de agrupamiento. Ello significa que tener un alto nivel de metamemoria no se traduce sin más en un mayor empleo de la estrategia de agrupamiento. Antes al contrario, puede generar la utilización de otras estrategias más eficaces. Esta interpretación viene avalada por la constatación de que los sujetos altos en metamemoria recuerdan más que los bajos, y sin embargo el grado de agrupamiento no difiere en ambos grupos. Parece, por tanto, lícito concluir que la mejora en el recuerdo tiene que deberse al empleo de otras estrategias más eficaces que la de organización.

Tomados globalmente, nuestros resultados invitan a continuar explorando la naturaleza de las relaciones entre la metamemoria y la memoria, que se han revelado más complejas de lo que cabría esperar en principio. Hay que elaborar instrumentos de medida de la metamemoria apropiados al nivel evolutivo del niño. Un cierto componente metamnémico puede ser crítico a una determinada edad y no resultar relevante en otra. En segundo lugar, hay que seguir perfeccionando los índices de evaluación de estrategias, de modo que resulten indicadores más precisos (sobre todo en el caso de la estrategia de agrupamiento) de las reglas organizativas que emplean los niños de diferentes edades. En este sentido, hemos advertido el carácter no acumulativo de los cambios en las estrategias mnémicas que se registra con la edad. También cabe la posibilidad de que el procedimiento empleado no haya sido sensible a diferencias temporales en el uso de esta estrategia. El índice ARC mide el agrupamiento de los ítems recordados, lo que puede no reflejar exactamente el empleo de una estrategia de este tipo en el momento de la memorización.

Por último, debemos señalar la necesidad de utilizar tareas de memoria que sobrepasen ligeramente las capacidades mnémicas de cada edad, de manera que exijan una evaluación ajustada de las demandas de la tarea y la selección de estrategias de memorización apropiadas.

No obstante, los resultados positivos obtenidos en los sujetos de segundo curso, donde las limitaciones señaladas no han tenido tanta influencia, son especialmente alentadores, dado que en la literatura se encuentran con alguna frecuencia hallazgos de ese tipo sólo con sujetos de más edad (Perlmutter, 1978; Waters, 1982). Ello confirma la conveniencia prioritaria de 
depurar metodológicamente las investigaciones, para estar en posición de construir un modelo teórico que las pueda integrar.

\section{Referencias}

BAINE, D. (1986): Memory and Instruction. Englewood Cliffs, New Jersey.

BelleZZA, F. S.,y Walker, R. J. (1974): "Storage-Coding Trade off in Short-Term Storew. Journal of Experimental Psychology, 102, 4, 629-633.

BJORK, R. A. (1975): "Short term storage: The ordered output of a central processor». En Restle, R. M. et al. (eds.): Cognitive Theory (Vol. 1). Hillsdale, N. J., Erlbaum.

Borkowski, J. G., y Cavanaugh, J. C. (1981): «Metacognition and Intelligence Theory». En Friedman, M. et al. (eds.): Intelligence and Learning. Nueva York, Plenum.

BORKOWSKI, J. G., et al. (1983): «Impulsivity and strategy transfer: Metamemory as mediator*. Child Development, 54, 459-473.

BROWN, A. L. (1978): «Knowing when, where and how to remember: A problem of metacognition». En Glaser, E. (ed.): Advances in instructional psychology. Hillsdale, N.J., Erlbaum.

BROW/N, A. L. (1980): «Metacognitive Development and Reading». En Spiro, R. J.; Bruce, B. C., y Brewer, W. F. (eds.): Theoretical Issuess in Reading Comprehension. LEA, Hillsdale, N. J.

BROWN, A. L., y LAWTON, S. C. (1977): «The feeling of knowing experience in educable retarded children». Developmental Psychology, 13, 364-370.

CAvANAUGH, J. C., y BORKOW/SKI, J. C. (1980): «Searching for metamemory-memory connections: A developmental study». Developmental Psychology, 16, 441-453.

Cavanaugh, J. C., y Perlmutter, M. (1982): Metamemory: A critical examination». Child Developmen, 53, 11-28.

Craik, F. I. M., y LOCKHART, R. (1972): «Levels of processing: A framework for memory research". Joumal of Verbal Learning and Verbal Behavior, 11, 671-684.

DiAZ, J. M., y RODRIGO, M. J. (en prensa): Metamemoria y memoria: un estudio evolutivo de sus relaciones funcionales.

ERICSSON, K. A., y SimON, H. A. (1980): Verbal reports as data. Psychology Review, 87, 215-251.

FlavelL, J. H. (1981): «Cognitive monitoring». En P. Dickson (ed.): Children's oral communication skills. Nueva York, Academic Press.

Flavell, J. H., y Wellman, H. M. (1977): "Metamemory». En Kail, R. V. y Hagen, J. W. (eds.): Perspectives on the development of memory and cognition. Hillsdale, N. J. Erlbaum.

GLASS, G. V. (1976): «Primary, secondary, and meta-analysis of research». Educational Researcher, 5, 3-8.

HAGEN, J. W. (1975): «Commentary». Monographs of the Society for Research in Child Development, 40 (1, Serial 159).

HerrmanN, D. J. (1982): «Knowing Thy Memory: The Use of Questionnaires to Assess and Study Memory. Psychological Bulletin, 92, 2, 434-452.

KOBASIGAWA, A. (1977): «Retrieval strategies in the development of memory *. En Kail, R. V. y Hagen, J. W. (eds.): Perspectives on the development of memory and cognition. Hillsdale, N. J. Erlbaum.

LANGE, G. (1978): «Organization-related processes in children's recall». En Ornstein, P. A. (ed.): Memory development in children. Hillsdale, N. J. Erlbaum.

LUNZER, E. A. (1979): "The development of consciousness». En Underwood, G. y Stevens, R. (eds.): Aspects of Consciousness. Nueva York, Academic Press.

PERLMUTTER, R. M. (1978): "What is memory aging the aging of? Developmental Psycho$\log y, 14,330-345$.

POSTMAN, L. (1978): «Picture-Word differences in the acquisition and retention of paired associates". Journal of Experimental Psychology, 4, 2, 146-157.

ROENKER, D. L.; ThOMPSON, C. P., y BROWN, S. C. (1971): *Comparison of Measures for the Estimation of Clustering in Free Recalls. Psychological Bulletin, 1976, 1, 45-48.

SCHNEIDER, W. (1985): *Developmental Trends in the Metamemory-Memory Behavior Relationship: An Integrative Review *. En Forrest-Presseley, D. L.; Mackinnon, G. E. y Gary Waller, T. (eds.): Metacognition, Cognition and Human Performance (Vol. 1), Academic Press, Orlando, Florida.

WATERS, H. S. (1982): «Memory development in adolescence: Relationships between metamemory, strategy use, and performance». Journal of Experimental Child Psychology, 33, 183-195.

WellmaN, H. M. (1977): «Tip of the tongue and feeling of knowing experience: A developmental study of memory monitoring». Child Development, 48, 13-21. 
Wellman, H. M. (1983): Metamemory revisited, en Chi, M. T. H. (ed.): Trends in memory development research. Basilea, Karger.

WIMMER, H, y TORNQUIST, K. (1980): *The role of metamemory and metamemory activation in the development of mnemonic performance». International Journal of Behavioral Development, 3, 71-81.

YUSSEN, S. R., y BERMAN, L. (1981): Memory predictions for recall and recognition in first-, third-, and fifth- grade children». Developmental Psychology, 17, 224-229.

\section{Extended summary}

This study attempts to evaluate the relationship between metamemory and mnemonic performance in two groups of children aged 7 and $14 . \mathrm{Li}-$ terature on the subject indicates the existence of certain conditioning factors in the relationship between both variables. Thus, the influence of methodological factors associated to the instrument used to evaluate the metamemory (e.g., based exclusively on verbal or non-verbal material) has been detected. From a theoretical point of view, no definitive contributions have been made, because models which attempt to provide an explanation of the pattern of the relationships between metamemory-memory are only tentative in nature. Furthermore, we can identify other sources of variation in such relationships, for example, the age of the subjects, the component of metamemory involved, and the level of difficulty of the task, are all present in such relations.

The present study focuses on two mnemonic strategies: sorting items according to their natural category, and codification. We designed a graphic-verbal task to evaluate metamemory. The task was given to two large groups of children aged 7 and 14 . We then selected the 10 children who had obtained the highest scores and the 10 children who had obtained the lowest scores in each age-group. To detect whether or not the classification strategy had been employed, we used the ARC index; and the of repetition-codification strategy was detected by means of an experimental variable (awareness, or lack of awareness, of the existence of a delayed memory task). Here we followed the reasoning of Bellezza and Walker (1974): those subjects who know that their recall in a latter task is going to be more rewarded than their immediate recall, devote part of their mnemonic resources to the storing of information in the LTM, and thus their immediate performance is diminished slightly. In order to achieve this, it is essential to use a repetition-codification strategy which, in turn, involves a certain level of sensitivity towards the differential requirements of the task.

In the analysis of results concerning the codification strategy, we can observe a recall pattern which is nearer to the one expected by 7 year old children than the 14 year old (in the latter group there is no difference between the children who have high and low metamemory). In the younger group, we recorded better performance in the case of those children with high metamemory. Furthermore, they prove to be (on the whole) more sensitive to being or not aware of the existence of the delayed memory task, all of which leads to a distribution more in keeping whith their mnemonic capacity. It is possible that the memory task used is more suitable for children aged 7 than for those aged 14, a fact which would account for the results obtained. 
As for the sorting strategy, again the two groups produced different results. In the older group, and contrary to what might be expected, the subjects with a low metamemory make greater use of this strategy, although this does not give rise to a better mnemonic performance. In the group of 7 years old, those subjects with high metamemory remember more, though they do not use the sorting strategy more frequently. All this indicates that they use other, more useful strategies.

Our results encourage further research on the metamemory-memory relationship, using instruments which are more sophisticated and also sensitive to the fact that one component of metamemory may be crucial at a certain age, but less important at a different age. In addition, memory tests suitable to each stage of evolution should be used, tests which put the subjects' mnemonic and metamnemonic resources to work, but without exceeding the limits of their capacity. 